\title{
Development of Optimal Denoising Technique using TV Regularization and Masking Filter
}

\author{
Vivek Kumar Sharma \\ M Tech Research Scholar, \\ OIST, Bhopal
}

\author{
Shreeja Nair \\ Research Guide, OIST, \\ Bhopal
}

\begin{abstract}
Image denoising is the fascinating research area among researchers due to applications of the images in everywhere, social networking sites, High Definition videos and stills. The need of it is to enhance the facility to imaging devices and the processing devices for denoising and enhancement of images. In this paper, Total Variation (TV) regularization is used to allow for accurate registration near such boundaries. We propose a novel formulation of TV-regularization for parametric displacement fields and Masking Filter to enhance or denoising of images. The proposed methodology's performance are usually compared in terms of peak-signal-tonoise ratio (PSNR). These are simply mathematically defined image metrics that take care of noise power level in the whole image.
\end{abstract}

\section{Keywords}

PSNR, Image Denoising, TV, Masking Filter.

\section{INTRODUCTION}

Digital Image Processing usually refers to the processing of a 2-dimensional (2-D) picture signal by a digital hardware. The 2-D image signal might be a photographic image, text image, graphic image (including synthetic image), biomedical image (X-ray, ultrasound, etc.), satellite image, etc. In a broader context, it implies processing of any 2-D signal using a dedicated hardware, e.g. an application specific integrated circuit (ASIC) or using a general-purpose computer implementing some algorithms developed for the purpose.

It is a hard task to distinguish between the domains of image processing and any other related areas such as computer vision. Though, essentially not correct, image processing may be defined as a process where both input and output are images. At the high level of processing and after some preliminary processing, it is very common to perform some analysis, judgment or decision making or perform some mechanical operation (robot motion). These areas are the domains of artificial intelligence (AI), computer vision, robotics.

Most of the image-processing functions are implemented in software. A significant amount of basic image processing software is obtained commercially.

\subsection{Major areas of image processing are:}

(i) Image Representation

(ii) Image Transformation

(iii) Image Enhancement

(iv) Image Restoration

(v) Color Image Processing

(vi) Transform-domain Processing

(vii) Image Compression (viii) Morphological Image Processing

(ix) Image Representation and Description

(x) Object Recognition

Image restoration is one of the prime areas of image processing and its objective is to recover the images from degraded observations. The techniques involved in image restoration are oriented towards modeling the degradations and then applying an inverse procedure to obtain an approximation of the original image. Hence, it may be treated as a deconvolution operation.

Depending on applications, there are various types of imaging systems. X-ray, Gamma ray, ultraviolet, and ultrasonic imaging systems are used in biomedical instrumentation. In astronomy, the ultraviolet, infrared and radio imaging systems are used. Sonic imaging is performed for geological exploration. Microwave imaging is employed for radar applications. But, the most commonly known imaging systems are visible light imaging. Such systems are employed for applications like remote sensing, microscopy, measurements, consumer electronics, entertainment electronics, etc.

\subsection{Order Statistics Filters}

Usually, sliding window technique is employed to perform pixel-by-pixel operation in a filtering algorithm. The local statistics obtained from the neighborhood of the center pixel give a lot of information about its expected value. If the neighborhood data are ordered (sorted), then ordered statistical information is obtained. If this order statistics vector is applied to a finite impulse response (FIR) filter, then the overall scheme becomes an order statistics (OS) filter.

\section{MEAN AND MEDIAN FILTERS}

In signal processing, it is often desirable to be able to perform some kind of noise reduction on an image or signal. The median filter is a nonlinear digital filtering technique, often used to remove noise. Such noise reduction is a typical preprocessing step to improve the results of later processing (for example, edge detection on an image). Median filtering is very widely used in digital image processing because, under certain conditions, it preserves edges while removing noise

The moving average or mean filter (MF) is a simple linear filter [1-6]. All the input data are summed together and then the sum is divided with the number of data. It is very simple to implement in hardware and software. The computational complexity is very low. It works fine for low power AWGN. As the noise power increases, its filtering performance degrades. If the noise power is high, then a larger window should be employed for spatial sampling to have better local statistical information. As the window size increases, MF produces a reasonably high blurring effect and thus thin edges and fine details in an image are lost. 


\subsection{Alpha Trimmed Mean Filter}

The alpha-trimmed mean (ATM) filter [1] is based on order statistics and varies between a median and mean filter. It is so named because, rather than averaging the entire data set, a few data points are removed (trimmed) and the remainders are averaged. The points which are removed are most extreme values, both low and high, with an equal number of points dropped at each end (symmetric trimming). In practice, the alpha-trimmed mean is computed by sorting the data low to high and summing the central part of the ordered array. The number of data values which are dropped from the average is controlled by trimming parameter alpha.

\section{PROPOSED METHODOLOGY}

The denoising is the necessity of the modern imaging solutions because these are the integrated part of human being from morning to evening round the clock. The every task has somewhere has the imaging devices from portable camera to smart phone camera to high end DSLRs, From Surveillance cameras to shooting of commercial videos, films, from scanners to fax, photocopiers all these devices need denoising mechanism to gives better and better results as their output performance.

In the same context this paper discussing about. The mechanism of denoising with efficient approach is described in this section. The block diagram and flow chart is shown in the below figures.

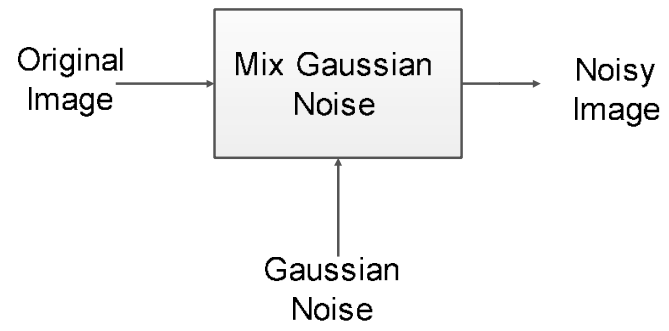

Fig.1 Process to Get Noisy Image

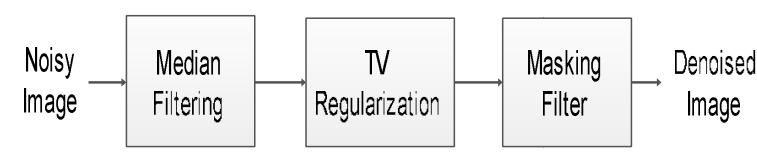

Fig 2: Block Diagram of Proposed Denoising System

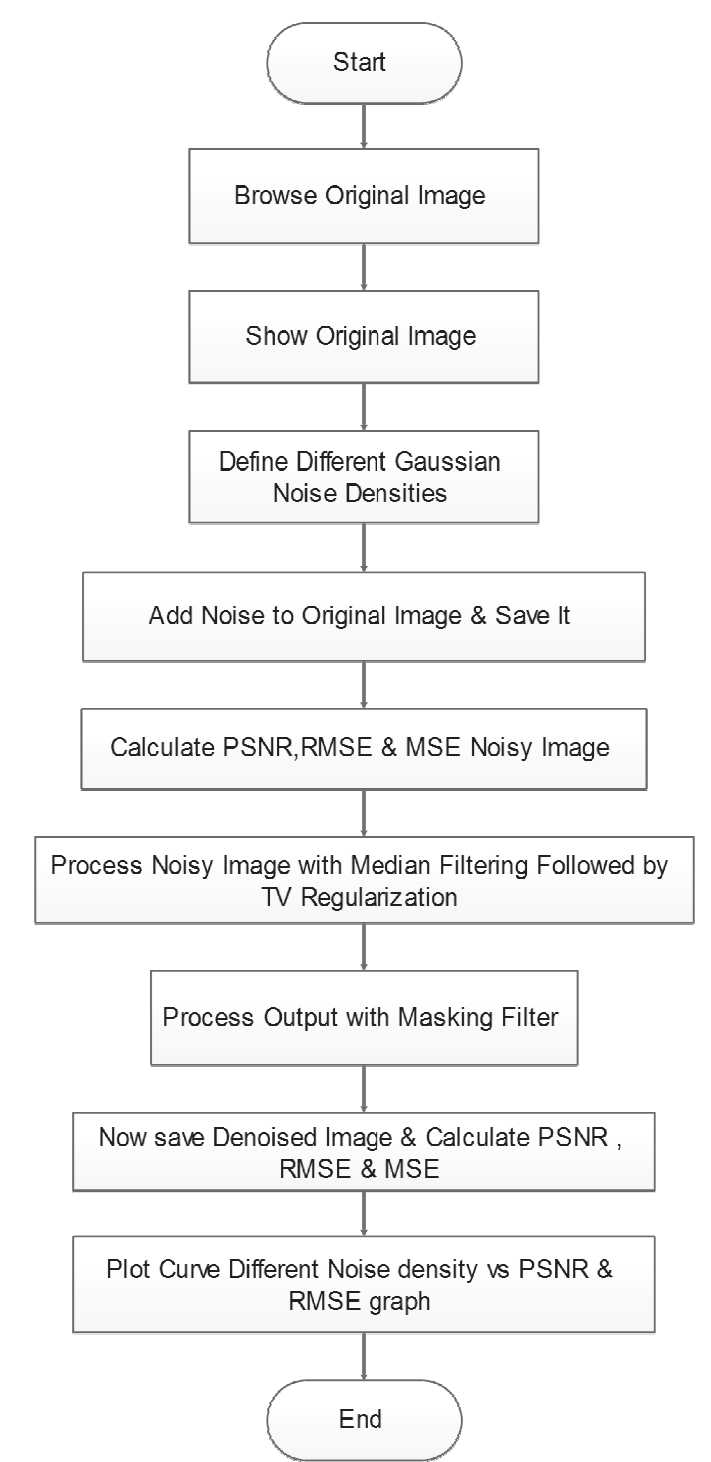

Fig 3: Flow Chart of the Proposed Denoising Algorithm

\section{SIMULATION OUTCOMES}

The previously discussed denoising system is implemented on MATLAB and the simulation outcomes are shown in below table with the help of images.

Below table shows the noisy and denoised image of Lena image for different noise densities with the peak signal to noise ratio (PSNR) and root mean square error (RMSE) of improved (denoised) results. 
Noisy Image

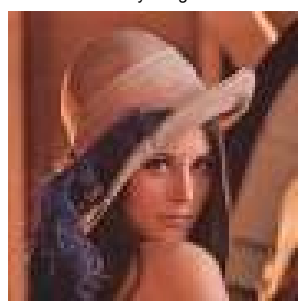

Noise Level $=0.01$

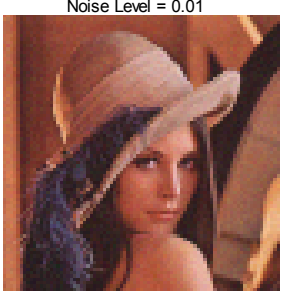

Noise Level $=0.10$

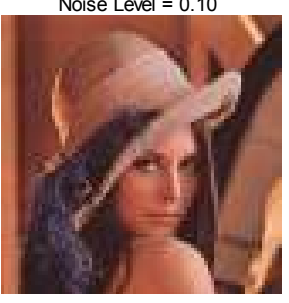

Noise Level $=0.02$

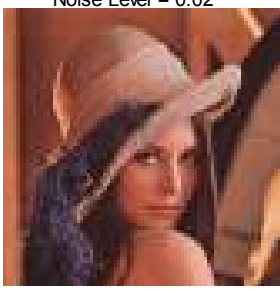

Noise Level $=0.03$

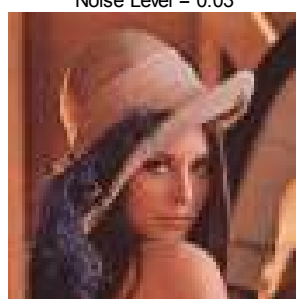

Noise Level $=0.04$

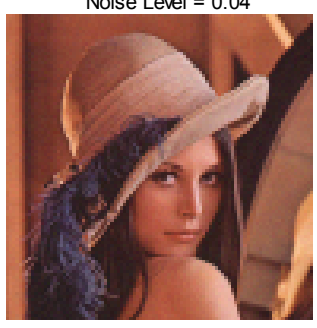

Noise Level $=0.05$
Denoised Image
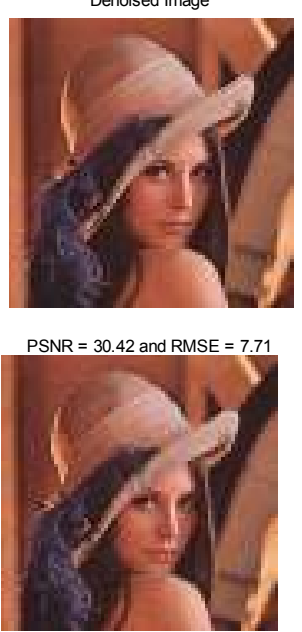

$\mathrm{PSNR}=26.77$ and $\mathrm{RMSE}=11.74$

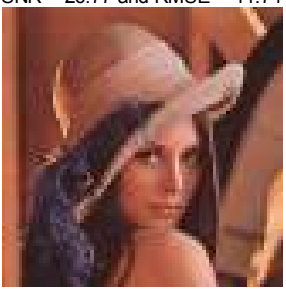

$\mathrm{PSNR}=30.33$ and $\mathrm{RMSE}=7.79$

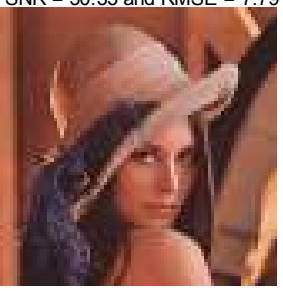

PSNR $=30.19$ and RMSE $=7.92$

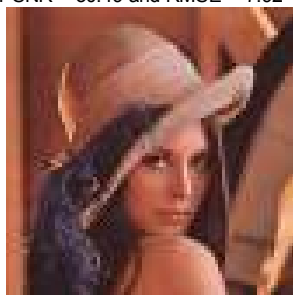

$\mathrm{PSNR}=30.01$ and $\mathrm{RMSE}=8.08$

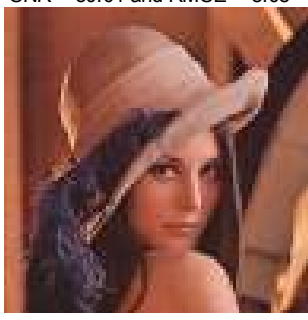

$\mathrm{PSNR}=29.72$ and $\mathrm{RMSE}=8.36$

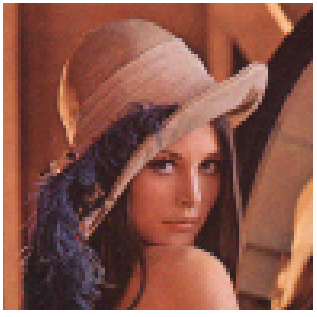

Noise Level $=0.06$

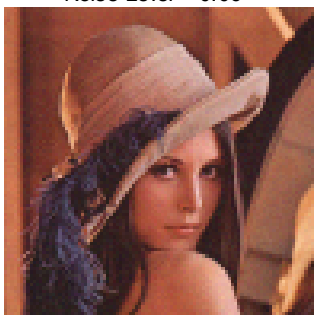

Noise Level $=0.07$

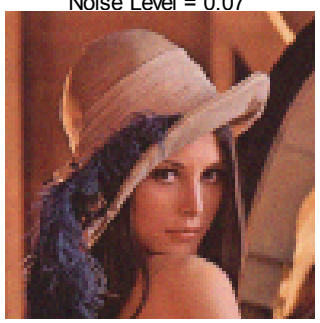

Noise Level $=0.08$

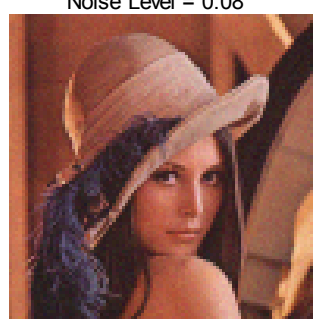

Noise Level $=0.09$

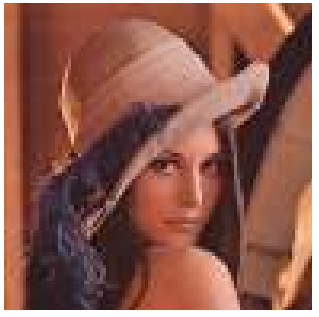

$\mathrm{PSNR}=29.32$ and $\mathrm{RMSE}=8.75$

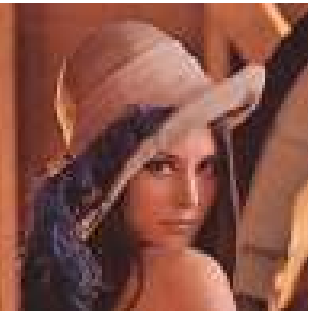

PSNR $=28.86$ and RMSE $=9.23$

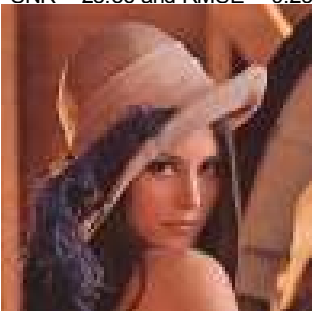

PSNR $=28.26$ and RMSE $=9.89$

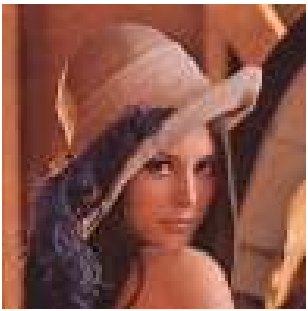

PSNR $=27.55$ and $\mathrm{RMSE}=10.74$

Fig 4: Lena Image Outcomes of Different Noise Levels left (Noisy Image), Right (Denoised Image and PSNR of it.

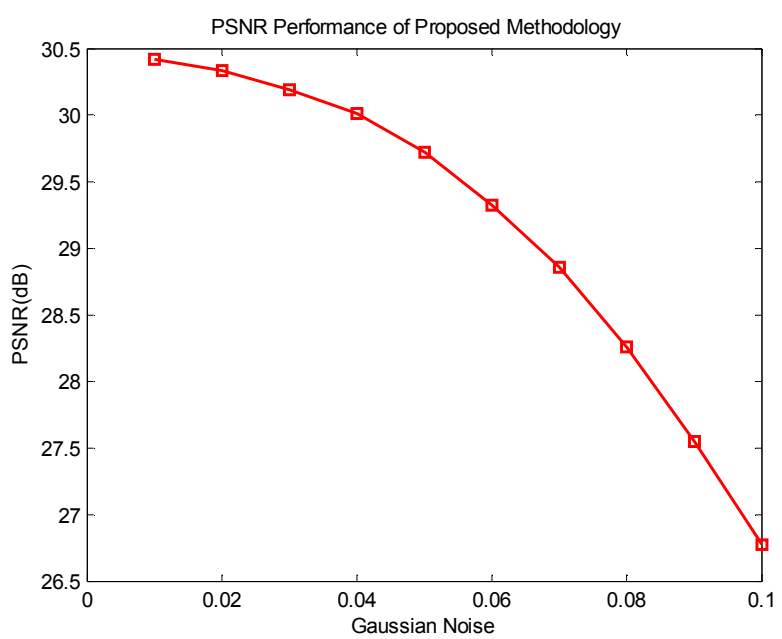

Fig 5: PSNR Curve of Lena Image for Different Noise Levels 


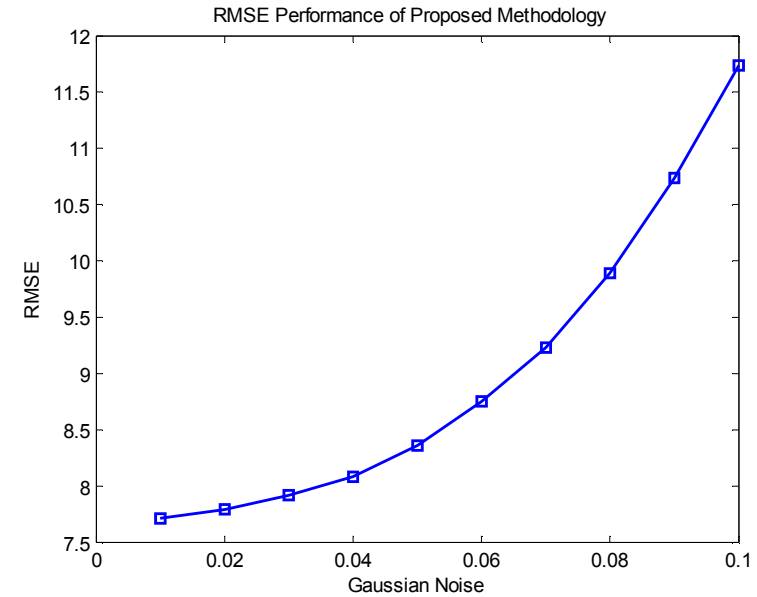

Fig 6: RMSE Curve of Lena Image for Different Noise Levels

The PSNR curve and RMSE curves are also shown in the above figures, which shows the performance of denoising system as noise increases.

Table 1. Comparison of PSNR

\begin{tabular}{|c|c|c|}
\hline \multirow{2}{*}{ Noise Level } & \multicolumn{2}{|c|}{ PSNR (dB) } \\
\cline { 2 - 3 } & Proposed & Previous \\
\hline 0.0100 & 30.4104 & 27.45 \\
\hline 0.0200 & 30.3400 & 27.09 \\
\hline 0.0300 & 30.1879 & 27.01 \\
\hline 0.0400 & 29.9941 & 26.78 \\
\hline 0.0500 & 29.7393 & 26.15 \\
\hline 0.0600 & 29.3307 & 21.69 \\
\hline 0.0700 & 28.8652 & 21.10 \\
\hline 0.0800 & 28.2574 & 20.51 \\
\hline 0.0900 & 27.5352 & 20.25 \\
\hline 0.1000 & 26.7526 & 20 \\
\hline
\end{tabular}

Table 2. Comparison of RMSE

\begin{tabular}{|c|c|c|}
\hline \multirow{2}{*}{ Noise Level } & \multicolumn{2}{|c|}{ RMSE } \\
\cline { 2 - 3 } & Proposed & Previous \\
\hline 0.0100 & 7.7218 & 17.11 \\
\hline 0.0200 & 7.7847 & 18.02 \\
\hline 0.0300 & 7.9221 & 20.22 \\
\hline 0.0400 & 8.1010 & 21.31 \\
\hline 0.0500 & 8.3421 & 22.5 \\
\hline 0.0600 & 8.7439 & 24.05 \\
\hline 0.0700 & 9.2253 & 25.02 \\
\hline 0.0800 & 9.8939 & 26 \\
\hline 0.0900 & 10.7517 & 27.1 \\
\hline 0.1000 & 11.7655 & 27.5 \\
\hline & & \\
\hline
\end{tabular}

Table 3. Comparison of Result Between reference Method and Proposed Method

\begin{tabular}{|c|c|c|c|}
\hline Image Level & Noise level & $\begin{array}{c}\text { Referrence } \\
\text { Method }\end{array}$ & $\begin{array}{c}\text { Proposed } \\
\text { Method }\end{array}$ \\
\hline Lena & 0.05 & 25.07 & 26.15 \\
\hline Subaru (car) & 0.05 & 23.75 & 26.05 \\
\hline Red Flower & 0.05 & 24.14 & 29.94 \\
\hline Beach & 0.05 & 22.97 & 27.95 \\
\hline
\end{tabular}

\section{CONCLUSION AND FUTURE SCOPES}

From the simulation outcomes it may be analyzed that the proposed denoising technique is far better than the previous system model. The comparison of PSNR and RMSE has been shown in Table 1 and Table 2 respectively that clearly shows the system which perform better for various imaging devices. Table 3 shows a comparison of PSNR result of reference method and proposed method. The wavelet transform is a transform it provides the time-frequency representation. Regularly a particular spectral component occurring at any instant may be of particular interest. In these cases it may be very beneficial to know the time intervals these particular spectral components occur. As the latency of an event-related potential is of particular interest, the latency of this response is the amount of time elapsed between the onset of the stimulus and the response. Wavelet transform is capable of providing the time and frequency information simultaneously, hence giving a time-frequency representation of the signal.

\section{REFERENCES}

[1] Kethwas, A.; Jharia, B., \& quot; Image de-noising using fuzzy and wiener filter in wavelet domain, \& quot; in Electrical, Computer and Communication Technologies (ICECCT), 2015 IEEE International Conference on , vol., no., pp.1-5, 5 March 2015.

[2] J. M. Parmar and S. A. Patil, "Performance evaluation and comparison of modified denoising method and the local adaptive wavelet image denoising method," Intelligent Systems and Signal Processing (ISSP), 2013 International Conference on, Gujarat, 2013, pp. 101-105.

[3] D. A. Huang, L. W. Kang, Y. C. F. Wang and C. W. Lin, "Self-Learning Based Image Decomposition With Applications to Single Image Denoising," in IEEE Transactions on Multimedia, vol. 16, no. 1, pp. 83-93, Jan. 2014.

[4] W. Liu and Z. Ma, "Wavelet Image Threshold Denoising Based on Edge Detection," Computational Engineering in Systems Applications, IMACS Multiconference on, Beijing, 2006, pp. 72-78.

[5] T. Shah, G. Shikkenawis and S. K. Mitra, "Epitome based transform domain Image Denoising," Advances in Pattern Recognition (ICAPR), 2015 Eighth International Conference on, Kolkata, 2015, pp. 1-6.

[6] B. S. Kim, M. S. Gil, M. J. Choi and Y. S. Moon, "Partial denoising boundary image matching using time-series matching techniques," 2015 International Conference on Big Data and Smart Computing (BIGCOMP), Jeju, 2015, pp. 136-141.

[7] E. Kugu, "Satellite image denoising using Bilateral Filter with SPEA2 optimized parameters," Recent Advances in Space Technologies (RAST), 2013 6th International Conference on, Istanbul, 2013, pp. 217-223.

[8] M. Thilagavathi and P. Deepa, "An efficient dictionary learning algorithm for 3d Medical Image Denoising based on Sadct," Information Communication and Embedded Systems (ICICES), 2013 International Conference on, Chennai, 2013, pp. 442-447.

[9] C. L. Tsai, W. C. Tu and S. Y. Chien, "Efficient natural color image denoising based on guided filter," Image Processing (ICIP), 2015 IEEE International Conference on, Quebec City, QC, 2015, pp. 43-47. 
[10] W. Liu, "New Method for Image Denoising while Keeping Edge Information," Image and Signal Processing, 2009. CISP '09. 2nd International Congress on, Tianjin, 2009, pp. 1-5.

[11] M. Ghazel, G. H. Freeman and E. R. Vrscay, "Fractalwavelet image denoising," Image Processing. 2002. Proceedings. 2002 International Conference on, 2002, pp. I-836-I-839 vol.1.

[12] X. Deng and Z. Liu, "An Improved Image Denoising Method Applied in Resisting Mixed Noise Based on MCA and Median Filter," 2015 11th International Conference on Computational Intelligence and Security (CIS), Shenzhen, 2015, pp. 162-166.
[13] X. Zeng, W. Bian, W. Liu, J. Shen and D. Tao, "Dictionary Pair Learning on Grassmann Manifolds for Image Denoising," in IEEE Transactions on Image Processing, vol. 24, no. 11, pp. 4556-4569, Nov. 2015.

[14] F. Chen, L. Zhang and H. Yu, "External Patch Prior Guided Internal Clustering for Image Denoising," 2015 IEEE International Conference on Computer Vision (ICCV), Santiago, 2015, pp. 603-611.

[15] M. G. McGaffin and J. A. Fessler, "Edge-Preserving Image Denoising via Group Coordinate Descent on the GPU," in IEEE Transactions on Image Processing, vol. 24, no. 4, pp. 1273-1281, April 2015. 